\title{
Efficacy and Safety of Washed Microbiota Transplantation to Treat Patients with Mild-to-Severe COVID-19 and Suspected of Having Gut Microbiota Dysbiosis: Study Protocol for a Randomized Controlled Trial*
}

\author{
Li-hao WU ${ }^{1,2 \dagger}$, Zhi-ning YE ${ }^{1,2 \dagger}$, Ping PENG $^{3}$, Wen-rui XIE ${ }^{1,2}$, Jia-ting XU ${ }^{1,2}$, Xue-yuan ZHANG ${ }^{1,2}$, Harry Hua-xiang XIA ${ }^{1}$, \\ Xing-xiang $\mathrm{HE}^{1,2 \#}$ \\ ${ }^{I}$ Department of Gastroenterology, the First Affiliated Hospital of Guangdong Pharmaceutical University, Guangzhou 510080, \\ China \\ ${ }^{2}$ Research Center for Engineering Techniques of Microbiota-Targeted Therapies of Guangdong Province, the First Affiliated \\ Hospital of Guangdong Pharmaceutical University, Guangzhou 510080, China \\ ${ }^{3}$ Guangzhou Eighth People's Hospital, Guangzhou 510060, China
}

(C) Huazhong University of Science and Technology 2021

\begin{abstract}
[Abstract] Objective: Coronavirus disease 2019 (COVID-19) is often accompanied by gastrointestinal symptoms, which are related to gut microbiota dysbiosis (GMD). Whether washed microbiota transplantation (WMT) is an effective treatment for COVID-19 patients suspected of having GMD by restoring the gut microbiota is unknown. This study is designed to explore the efficacy and safety of WMT in COVID-19 patients suspected of having GMD. Methods: This is a randomized, multicenter, single-blind prospective study. COVID-19 patients suspected of having GMD will be randomly divided to receive routine treatment only or to receive routine treatment and WMT. The frequency of WMT will be once a day for three consecutive days. Laboratory and imaging examinations will be performed at admission, 1 and 2 weeks after treatment, and on the day of discharge. Then a telephone follow-up will be conducted at 1 st week, 2 nd week, and 6 th month after discharge. The clinical efficacy and safety of WMT in COVD-19 patients suspected of having GMD and the effects of WMT on the organ function, homeostasis, inflammatory response, intestinal mucosal barrier function, and immunity of the patients will be evaluated. Results: By following the proposed protocol, WMT is expected to be efficacious and safe for the treatment of COVID-19 patients suspected of having GMD, and the therapeutic effect is expected to be associated with improvement of the intestinal mucosal barrier function, inflammatory response, and immunity. Conclusion: The findings from this study may offer a new approach for the prevention and treatment of COVID-19 patients suspected of having GMD.
\end{abstract}

Key words: COVID-19; SARS-Cov-2 virus; gut microbiota; microbiome; dysbiosis; washed microbiota transplantation

Coronavirus disease 2019 (COVID-19), caused by the SARS-Cov-2 virus, has become a worldwide pandemic. On January 30, 2020, the World Health Organization announced the outbreak of COVID-19 as

Li-hao WU, E-mail: wulihao888@126.com; Zhi-ning YE, E-mail: 1554520250@qq.com

"Li-hao WU and Zhi-ning YE contributed equally to this work.

\#Corresponding author, E-mail: hexingxiang@gdpu.edu.cn ${ }^{*}$ This study was supported by the Special Research Project on Prevention and Control of COVID-19 in Universities of the Guangdong Provincial Education Department (No. 2020KZDZX1132).

Electronic supplementary material The online version of this article (https://doi.org/10.1007/s11596-021-2475-

2) contains supplementary material, which is available to authorized users. a public health emergency of international concern ${ }^{[1]}$. By October 22, 2021, SARS-Cov-2 had infected more than 240 million people, with more than 4 million deaths worldwide (https://covid19.who.int/, October $22,2021)$. A meta-analysis has demonstrated that $17.6 \%$ of patients with SARS-Cov-2 infection developed gastrointestinal symptoms ${ }^{[2]}$. In addition, Zuo et al found that the gut microbiota changed significantly in COVID-19 patients, which manifested due to the increased abundance of opportunistic pathogens such as Coprobacillus, Clostridium ramosum, and Clostridium hathewayi and the decreased abundance of Faecalibacterium prausnitzii (an anti-inflammatory bacterium) and other bacteria that downregulate the expression of angiotensin-converting enzyme 2 (ACE2; the receptor of SARS-Cov-2), such as Bacteroides dorei, Bacteroides thetaiotaomicron, Bacteroides 
massiliensis, and Bacteroides ovatus ${ }^{[3]}$.

A previous animal study has shown that the imbalance of the gut microbiota resulted in the release of lipopolysaccharide (LPS) into the blood, leading to the upregulation of toll-like receptor 4 (TLR4), which is activated by influenza virus ${ }^{[4]}$. The excess activated TLR4 induced a cytokine storm through the TLR4nuclear factor-kappa B pathway, and the uncontrolled inflammatory response ultimately led to multiple organ failure in COVID-19 patients $^{[4,5]}$. A recent animal study also found that the normal gut microbiota composition could effectively reduce the early replication of influenza virus in lung epithelial cells ${ }^{[6]}$. Another animal study further demonstrated that restoration of the gut microbiota balance with probiotics or fecal microbiota transplantation (FMT) treatment significantly reduced influenza virus shedding and restored the expression level of interleukin (IL)-22, thus improving the survival of patients with lethal influenza ${ }^{[7]}$. Furthermore, Lee et al have found that the duration of viral shedding in COVID-19 patients was negatively correlated with the expression of the T-cell cytokines IL- $1 \beta$ and IL-17A ${ }^{[8]}$. Therefore, restoration of the gut microbiota balance seems to be an effective way to reduce the shedding of SARS-Cov-2 by regulating the body's immune response.

d'Ettorre et al have found that the supplementation of probiotics to routine treatment significantly improves the gastrointestinal symptoms and other clinical symptoms of COVID-19 patients $^{[9]}$. However, the role of probiotics in microbiome reconstruction is generally recognized to be not as effective as FMT. The improved methodology of FMT based on an automatic washing process and the related delivery method was coined as washed microbiota transplantation (WMT) by the consensus statement from the FMTstandardization Study Group in 2019 ${ }^{[10,11]}$. Importantly, whether WMT has therapeutic effects on COVID19-related gut dysbiosis is unknown. In this clinical trial protocol, WMT will be employed to treat gut microbiota dysbiosis (GMD). We designed this clinical trial protocol to explore the clinical efficacy and safety of WMT on COVID-19 patients suspected of having GMD.

\section{MATERIALS AND METHODS}

\subsection{Participants}

According to the COVID-19 diagnosis and treatment guidelines in China (7th edition), patients with positive COVID-19 nucleic acid tests (as detected by using a nasopharyngeal swab test) should be diagnosed as positive cases, which are required to be hospitalized and given the specific treatment according to the condition of the patient ${ }^{[12]}$. Patients diagnosed with COVID-19 can be divided into mild, moderate, severe, and critical types. The mild type is defined when the clinical symptoms are mild, without any imaging changes. The moderate type is defined when the patients have clinical symptoms such as fever, respiratory tract infection, etc., with pulmonary imaging changes. The severe type is defined when the patients meet any of the following conditions: the respiratory rate $>30$ breaths/ min, the pulse oxygen saturation $\leq 93 \%$, and the ratio of arterial oxygen partial pressure to fractional inspired oxygen $\leq 300 \mathrm{mmHg}$. The critical type is defined when the patients meet any of the following conditions: respiratory failure, requiring mechanical ventilation, shock, other organ failures and being treated in ICU.

Patients diagnosed with mild-to-severe COVID-19 and suspected of having GMD will be recruited for this clinical trial. Patients suspected of having GMD are defined as those with gastrointestinal symptoms (e.g., abdominal pain, heartburn, acid regurgitation, sucking sensations in the epigastrium, nausea and vomiting, borborygmus, abdominal distension, eructation, increased flatus, decreased passage of stools, increased passage of stools, loose stools, hard stools, urgent need for defecation, and feeling of incomplete evacuation) or antibiotic-associated diarrhea.

Patients with any of the following criteria will be excluded: critical type of COVID-19 according to the COVID-19 diagnosis and treatment guidelines in China (7th edition) $)^{[12]}$, gastrointestinal perforation, gastrointestinal obstruction, recent history of intestinal fistula, disturbance of consciousness, gastrointestinal hemorrhage, malignant tumors, and pregnant or lactating women.

In addition, the criteria for case rejection, dropout, and discontinuation in this study include the following: patients with poor compliance and unable to receive WMT, which is defined as an inability to cooperate with endoscopic catheterization, an inability to receive fecal suspension injections, or an inability to cooperate with follow-up; patients who participate in other trials or cannot follow the routine treatment during the present trial; patients with WMT-related adverse events that need to be treated; and patients with an aggravated illness or severe complications during the trial that must be immediately managed.

The clinical trial protocol was evaluated and approved by the Ethics Review Committee of the First Affiliated Hospital of Guangdong Pharmaceutical University. If any content of the protocol is changed, the researcher will submit the updated version of the protocol to the Ethics Review Committee of the First Affiliated Hospital of Guangdong Pharmaceutical University. After approval by the Ethics Review Committee of the First Affiliated Hospital of Guangdong Pharmaceutical University, the researcher will upload the updated protocol to the Chinese Clinical Trial Registry [Trial Registration: Chinese 
Clinical Trial Registry-URL: http://www.chictr.org. $\mathrm{cn} /$ showproj.aspx?proj $=53458$. Registration number: ChiCTR2000032737. Registered 9 May 2020].

A written informed consent form will be obtained from all participants. This trial will be conducted based on the principles of the Declaration of Helsinki (Fortaleza, 2013) and the International Ethical Guidelines for Research Involving Human Health (Geneva, 2016).

\subsection{Interventions}

This is a randomized, multicenter, single-blind, prospective study with a 1:1 allocation ratio. Eligible patients will be randomly divided into group A, who will receive routine treatment following the COVID-19 diagnosis and treatment guidelines in China (7th edition) ${ }^{[12]}$, and group B, who will receive WMT with routine treatment. The frequency of WMT will be once a day for three consecutive days.

\subsection{Laboratory and Clinical Evaluations}

The laboratory and clinical indicators will be tested or recorded at admission, at the 1 st and 2 nd week after treatment, and on the day of discharge. The laboratory indicators will include a nasopharyngeal swab test, feces SARS-Cov-2 RNA test, fasting blood glucose, muscle enzyme, D-dimer, electrolytes, myoglobin, C-reactive protein (CRP), erythrocyte sedimentation rate (ESR), procalcitonin (PCT), peripheral blood lymphocyte subsets, oxygenation index, blood gas analysis, routine blood tests, liver function indicators, renal function indicators, inflammatory cytokines such as interleukin (IL)-2, IL-6, tumor necrosis factor alpha (TNF- $\alpha$ ), interferon-gamma (IFN- $\gamma$ ), and intestinal mucosal barrier function-related factors, such as LPS, D-lactate, and diamine oxidase (supplementary table 1). All samples will be collected by a clinical physician. Arterial blood samples will be used for blood gas analysis and determination of oxygenation indexes with a blood gas analyzer. Venous blood samples will be used for the detection of the remaining indicators with a Sysmex 1000i blood cell analyzer, Cobas501 automatic biochemical analyzer, and JYDLT intestinal mucosal barrier function analysis system, respectively. In addition, the nasopharyngeal swabs and fecal samples will be analyzed by KingMed Diagnostics (China).

The clinical indicators will include fever, pulmonary shadow absorption, gastrointestinal symptoms, etc. (supplementary table 2). In addition, all known WMT-related adverse events such as abdominal pain, abdominal distention, diarrhea, etc. will be monitored. All patients will be graded with the Pneumonia Severity Index (PSI, a scale to assess the severity of community-acquired pneumonia) ${ }^{[13]}$ (supplementary table 3) and Acute Physiology and Chronic Health Evaluation II (APACHE II, a classification system to assess disease severity ${ }^{[14]}$ (supplementary table 4).

\subsection{Recruitment Strategy}

All patients included in this study will be admitted to Guangzhou Eighth People's Hospital, which is mainly responsible for the centralized treatment of COVID-19 patients in Guangdong Province and receive intervention treatment there. Researchers will recruit the qualified patients through clinical physician and recruitment advertisements (online or offline advertising). The recruitment period will last until June 2021. Ping PENG will be responsible for recruiting patients and obtaining informed consent when the patients are hospitalized.

\subsection{Study Unit and Group Composition}

This trial will be completed jointly by the First Affiliated Hospital of Guangdong Pharmaceutical College and Guangzhou Eighth People's Hospital. The First Affiliated Hospital of Guangdong Pharmaceutical College will be responsible for the preparation of the stool suspension and the operation of WMT, and Guangzhou Eighth People's Hospital will be responsible for collecting all of the data listed above. All the data will be analyzed mutually by the biostatisticians participating in this study.

The coordinating group, composed of Wen-rui XIE and Jia-ting XU, is responsible for coordinating the work of researchers at Guangzhou Eighth People's Hospital and the First Affiliated Hospital of Guangdong Pharmaceutical University, respectively.

The steering committee, composed of Li-hao WU, Ping PENG, and Xing-xiang HE, is mainly responsible for guiding the development and distribution of research work. The steering committee will organize a monthly trial meeting of the coordination group, the endpoint adjudication committee, the data management team, and the ethics committee for auditing trial conduct during the month. The endpoint adjudication committee will be represented by the Ethics Committee of the First Affiliated Hospital of Guangdong Pharmaceutical University, which does not include participants in this study and is mainly responsible for the adjudication of endpoint events. The data management team, composed of Er-hua ZHANG, Zhi-he DENG, Zhi-ning YE, and Yu-pei ZHUANG, will be responsible for data collection, entry, and analysis during the study.

\subsection{Randomization and Blinding}

The enrolled patients will be allocated into group A and group B by the block randomization method. Taking the admission time as the compatibility factor, two patients with an adjacent admission time will be taken as a block group. The first patient in each block group will get a random number by querying a random number table. If the random number is odd, the patient will be included in group A, and the remaining patient in the block group will be included in group B. If the random number is even, the patient will be included in group $\mathrm{B}$, and the remaining patient in the block group 
will be included in group A. The randomization will be completed by a staff member (nurse or clerk) who is not in the research group. After the assignment, the staff member will put the results in an opaque sealed envelope. After each patient enters the trial, Ping PENG will open the envelope and carry out the corresponding intervention for the patient.

A single-blinded method will be used in this study. We will implement a blinding method for researchers in this study. One or more staff members will be selected to be responsible for recording the clinical data and testing the experimental samples for all enrolled patients, but they will not be admitted to access the information regarding which treatment the patients have received or which group the patients belong to.

\subsection{WMT}

1.7.1 Stool Donors The methods for donor screening and stool suspension preparation will be consistent with the established guidelines of WMT ${ }^{[11,15]}$. COVID-19 patients and close contacts of COVID-19 patients will be excluded in the screening of stool donors. Donors with typical COVID-19 symptoms (e.g., fever, fatigue, dry cough, myalgia, dyspnea, and headache) within the previous 30 days will not be approved to donate stools. In addition, donors will be asked to provide evidence such as big data travel card and health code confirming that they have not visited an area known to be affected by a COVID-19 outbreak or have a history of close contact with a confirmed or suspected COVID-19 patient within the previous 30 days. Only donors with a negative nasopharyngeal swab test within 7 days will be approved to donate stools.

The age of the donors will be between 18 and 25 years old, and their body mass index will be between 18.5 and 23.9. All donors first need to pass the questionnaire, and those who meet the requirements will receive a further interview, psychological examination, and physical examination. Donors with any of the following diseases will be excluded: infectious disease, digestive disease, metabolic disease, chronic fatigue syndrome, autoimmune disease, allergic disease, and neuropsychiatric disease. All qualified donors will receive the training required, such as information on a healthy diet, and then donate their stool sample in consultation with researchers.

1.7.2 Stool Suspension The stool samples provided by the donor will be collected, weighed, added to sterile saline in a 1:5 ratio of feces to saline, and then mixed evenly. The mixture will be filtered through an intelligent microbial separation system (GenFMTer; FMT Medical, China), and then five stages of filtration will be carried out. The obtained suspension will be immediately centrifuged at a speed of $2500 \mathrm{r} / \mathrm{min}$ for $3 \mathrm{~min}$, and this step will be repeated three times. The final sediment will be suspended again with sterile saline at a 1:1 ratio of sediment to saline.
1.7.3 WMT Preoperative Preparation Metoclopramide will be injected intramuscularly at $30 \mathrm{~min}$ before the WMT, and a proton pump inhibitor will be injected intravenously at $1 \mathrm{~h}$ before the WMT.

1.7.4 WMT Procedure A nasojejunal tube will be placed in the middle digestive tract. Normal saline will be used to flush the tube first, followed by the infusion of the stool suspension with the same volume of $200 \mathrm{~mL}$ per person. After the WMT, the patient will be asked to stay in the lying position for $30 \mathrm{~min}$, with restriction of strenuous activities.

\subsection{Participant Rights and Confidentiality}

The participants have the right to withdraw from the study freely at any stage of the study without discrimination or retaliation as well as have their medical rights unaffected. All of the information collected in this study will belong to the private information of the patients, and none of the researchers will be allowed to disclose the information unless they obtain permission from the patients. Codes will be used to identify the participants in all research-related documents. Only the research teams and sponsors, ethics committees, and regulatory agencies have access to the code information and other research materials, except for the participants' personal information and related information. The research results will be allowed to be published in relevant scientific journals, but the participants' names will not appear in any research reports or public publications.

\subsection{Outcomes}

1.9.1 Primary Outcome The primary outcome of this study will be the clinical efficacy of WMT on COVID-19 patients suspected of having GMD. The clinical efficacy will be reflected by the SARS-Cov-2 infection status and the recovery of the disease, which will be defined as "improvement" (from the severe type to the moderate or mild type, or from the moderate type to the mild type). In addition, the improvement of gastrointestinal symptoms will be a co-primary outcome.

1.9.2 Secondary Outcomes The following secondary outcomes will be assessed: 1) advantages of WMT over routine treatment, as reflected by the recovery time (i.e., the time from positivity to negativity by the nasopharyngeal swab test; the clearance time of SARS-Cov-2 from feces, defined as the time from positivity to negativity by the feces SARS-Cov-2 RNA test; the time from occurrence to improvement or even disappearance of various clinical symptoms; and the time from hospitalization to discharge), PSI grade, and APACHE II score; 2) effects of WMT on organ functions and homeostasis, as reflected by fasting blood glucose, muscle enzyme, D-dimer, electrolytes, myoglobin, liver function, and renal function; 3) effects of WMT on the inflammatory response, as reflected by routine blood tests, CRP, ESR, PCT, and 
inflammatory cytokines such as IL-2, IL-6, TNF- $\alpha$, and IFN- $\gamma$; 4) effects of WMT on intestinal mucosal barrier function, as reflected by LPS, D-lactate, and diamine oxidase levels; 5) effects of WMT on cellular immune functions, as reflected by the peripheral blood lymphocyte subsets; 6 ) the safety of WMT, as reflected by WMT-related adverse events.

In addition to the analysis of the differences between group A and group B, we also conduct subgroup analyses according to the severity of COVID-19 (including the mild, moderate, and severe groups), which analyzes the main and secondary outcomes.

\subsection{Follow-up Visit}

During hospitalization, the researchers will be asked to record the patients' clinical symptoms and signs, WMT-related adverse events, and treatment information every day. On the first day after randomization, the above laboratory and imaging examinations will be completed and then reviewed once a week. In addition, the PSI score and APACHE II grade will be estimated at the same time as completing these examinations. After discharge, telephone followup interviews will be conducted at 1st week, 2nd week, and 6th month after discharge, and the contents will include the clinical symptoms and signs listed above (mainly the symptoms and signs related to COVID-19 or WMT treatment) (supplementary table 5).

The schedule of enrollment, interventions, assessments, and follow-up is shown in fig. 1 .

\begin{tabular}{|c|c|c|c|c|c|c|c|c|c|c|}
\hline \multirow[b]{3}{*}{ TIMEPOINT $^{* *}$} & \multirow{3}{*}{$\begin{array}{c}\text { Enrolment } \\
0\end{array}$} & \multirow{3}{*}{$\begin{array}{c}\text { Allocation } \\
0\end{array}$} & \multicolumn{8}{|c|}{ Study period } \\
\hline & & & \multicolumn{7}{|c|}{ Post-allocation } & \multirow{2}{*}{$\begin{array}{c}\text { Close-out } \\
6 \mathrm{~W} \text { after } \\
\text { discharge }\end{array}$} \\
\hline & & & $1 \mathrm{D}$ & $2 \mathrm{D}$ & $3 \mathrm{D}$ & $1 \mathrm{~W}$ & $2 \mathrm{~W}$ & $\begin{array}{l}1 \mathrm{~W} \text { after } \\
\text { discharge }\end{array}$ & $\begin{array}{l}2 \mathrm{~W} \text { after } \\
\text { discharge }\end{array}$ & \\
\hline \multicolumn{11}{|l|}{ ENROLMENT: } \\
\hline Eligibility screen & $\mathrm{X}$ & & & & & & & & & \\
\hline Informed consent & $\mathrm{X}$ & & & & & & & & & \\
\hline Allocation & & $\mathrm{X}$ & & & & & & & & \\
\hline \multicolumn{11}{|l|}{ INTER VENTIONS: } \\
\hline [Routine treatment] & & & 4 & & & & $\rightarrow$ & & & \\
\hline$[\mathrm{WMT}]$ & & & 4 & & $\rightarrow$ & & & & & \\
\hline \multicolumn{11}{|l|}{ ASSESSMENTS: } \\
\hline $\begin{array}{r}\text { [Laboratory indicators } \\
\text { (nasopharyngeal swab } \\
\text { test, feces SARS-Cov-2 } \\
\text { RNA test, fasting blood } \\
\text { glucose, muscle enzyme, } \\
\text { D-dimer, electrolytes, } \\
\text { myoglobin, CRP, ESR, } \\
\text { PCT, peripheral blood } \\
\text { lymphocyte subsets, } \\
\text { oxygenation index, } \\
\text { blood gas analysis, } \\
\text { routine blood tests, liver } \\
\text { function test, renal } \\
\text { function test, IL-2, IL-6, } \\
\text { TNF- } \alpha, \text { IFN- }-, \text { LPS, } \\
\text { D-lactate diamine } \\
\text { oxidase)] }\end{array}$ & $\mathrm{X}$ & & $\mathrm{X}$ & & & $\mathrm{X}$ & $\mathrm{X}$ & & & \\
\hline $\begin{array}{r}\text { [Clinical indicators } \\
\text { (fever, pulmonary } \\
\text { shadow absorption, } \\
\text { gastrointestinal } \\
\text { symptoms,etc.)] }\end{array}$ & $\mathrm{X}$ & & $\mathrm{X}$ & & & $\mathrm{X}$ & $\mathrm{X}$ & $\mathrm{X}$ & $\mathrm{X}$ & $\mathrm{X}$ \\
\hline $\begin{array}{r}\text { [WMT related adverse } \\
\text { events (abdominal pain, } \\
\text { abdominal distention, } \\
\text { diarrhea, etc.)] }\end{array}$ & & & & & & & & & & \\
\hline
\end{tabular}

Fig. 1 Schedule of enrollment, interventions, assessments, and follow-up

D, day; W, week; M, month; WMT, washed microbiota transplantation; CRP, C-reactive protein; ESR, erythrocyte sedimentation rate; PCT, procalcitonin; IL-2, interleukin-2; IL-6, interleukin-6; TNF- $\alpha$, tumor necrosis factor alpha; IFN- $\gamma$, interferon gamma; LPS, lipopolysaccharide 


\subsection{Statistical Analysis}

The measurement data that conform to normal distribution will be expressed as the mean \pm standard deviation (SD), and those do not conform to normal distribution will be expressed as the median \pm interquartile range. If a normal distribution is obeyed, the Student's $t$-test will be used for the data of two groups, and analysis of variance will be used for the data of multiple groups. If there is a statistical difference between the groups, the least significant difference test will be used for pairwise comparison. If a normal distribution is not obeyed, the KruskalWallis $H$ test will be used for the comparison between the groups. The enumeration data will be expressed as cases (percentages) and tested by the chi-squared test or Fisher's exact test. If there is a statistical difference among the groups, the partitioned chi-squared test will be used for pairwise comparison. In addition, bivariate analysis will be performed for all variables, where variables with $P$ values $\leq 0.20$ will be analyzed by multivariate logistic regression. If a patient drops out of the study due to abandoning the intervention or failing to cooperate with the follow-up, we will ask the patient if he or she agrees to the use of the data. If the patient's consent is obtained, the multiple imputation method will be used to fill in the missing data. SPSS software version 19.0 (IBM, USA) will be used to analyze the data. Two-sided tests will be selected based on expertise, and $P<0.05$ will be defined as statistically significant.

\section{RESULTS}

\subsection{Primary Outcomes}

As compared with group A, it is expected that COVID-19 will be significantly improved in group B, with the negative conversion of SARS-Cov-2 virus and alleviated or disappeared gastrointestinal symptoms (fig. 2). These expected findings suggest that WMT is an effective treatment for COVID-19 patients suspected of having GMD.

\subsection{Secondary Outcomes}

The following secondary outcomes are expected: 1) the recovery time will be shorter and PSI grade and APACHE II scores (fig. 2) will be lower in group B than in group A. These expected findings suggest that the addition of WMT to routine treatment is beneficial for the recovery of COVID-19 patients suspected of having GMD. 2) The values of fasting blood glucose, muscle enzyme, D-dimer, electrolytes, myoglobin, liver function, and renal function will be restored to the levels closer to normal values and in a faster way in group B than in group A. The difference is expected to be statistically significant. These expected findings suggest that WMT contributes to maintaining the organ function and homeostasis. 3) The values of inflammatory indicators, including routine blood tests, CRP, ESR, PCT, IL-2, IL-6, TNF- $\alpha$, and IFN- $\gamma$, will be restored to the levels closer to normal values and in a faster way in group B than in group A. The differences are expected to be statistically significant. These expected findings indicate that WMT can effectively alleviate systemic inflammation and avoid the lung injury caused by a cytokine storm. 4) The values of intestinal barrier mucosal function-related factors such as LPS, D-lactate, and diamine oxidase will be restored to the levels closer to normal values and in a faster way in group B than in group A. The difference is expected to be statistically significant. These expected findings imply that the intestinal mucosal barrier function in COVID-19 patients can be repaired after WMT. 5) The peripheral lymphocyte subsets of COVID-19 patients gradually tend to get normal in both groups, which will be faster in group A than in group B. These expected findings indicate that WMT can effectively improve the abnormal immune activation and immune damage caused by SARS-Cov-2 infection. 6) The frequency of adverse events will be similar between the two groups or higher in group B than in group A, but the adverse events are all expected to be mild, with no or rare serious adverse events in group B. These expected findings show that WMT is safe for COVID-19 patients suspected of having GMD (fig. 2).

\section{DISCUSSION}

In the proposed clinical trial, WMT plus routine treatment is expected to be more efficacious than the routine treatment as well as safe for COVID-19 patients suspected of having GMD. The clinical efficacy is anticipated to be associated with improvement of the intestinal mucosal barrier function, inflammatory response, and immunity in patients.

SARS-Cov-2 virus is so closely related to the digestive system that it can easily survive in the patient's digestive tract and cause gastrointestinal symptoms $^{[2,16]}$. It has been revealed that there are antagonistic or coexisting relationships among various gut microorganisms ${ }^{[17]}$, and SARS-Cov-2 virus, as a new member, is bound to disrupt the balance of the gut microbiota ${ }^{[3]}$. Therefore, the correction of GMD may play a special role in the recovery of COVID-19 patients. WMT has been proved to be a safe method with considerable clinical efficacy and negligible adverse events, particularly in the treatment of gastrointestinal disorders caused by GMD ${ }^{[18]}$. A previous study showed that probiotics or WMT interventions for highly pathogenic influenza virus infection can restore IL-22 to normal levels and improve the ability of animals to resist influenza virus infection ${ }^{[7]}$. However, there have not been any clinical trials to confirm this observation in humans. 


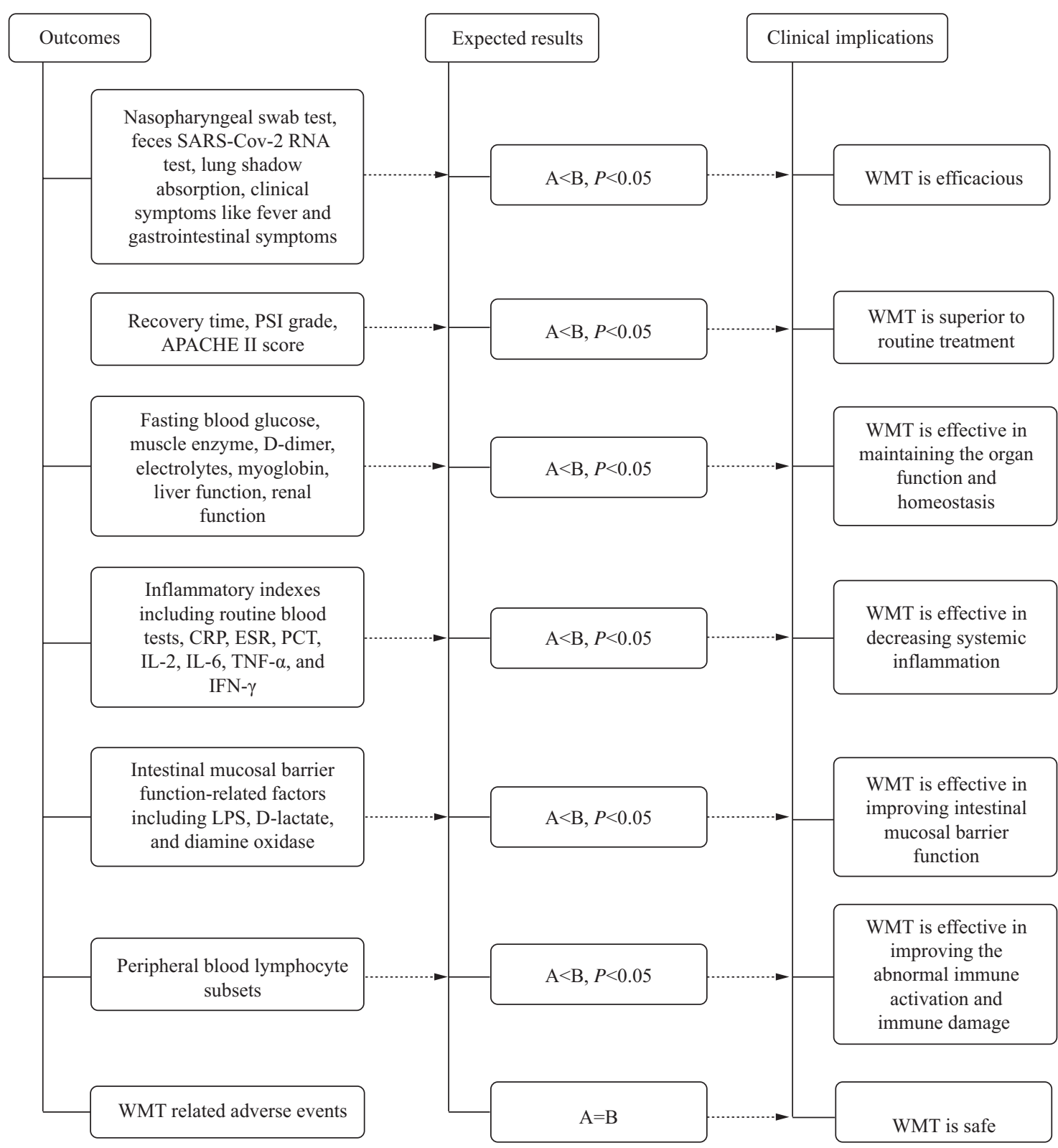

Fig. 2 Primary and secondary outcomes, expected results, and their clinical implications in the proposed study

The patients in group A will be treated with routine treatment, and those in group B will be treated with WMT and routine treatment. " $\mathrm{A}=\mathrm{B}$ " means that the value of group $\mathrm{A}$ is not different from that of group $\mathrm{B}$ for the same observation indicators. " $\mathrm{A}<\mathrm{B}$ " means that the values of the same observation indicators in group $\mathrm{B}$ are closer to normal values than those in group A, and the recovery time is shorter than that in group A. A, group A; B, group B; WMT, washed microbiota transplantation; PSI, Pneumonia Severity Index; APACHE II , Acute Physiology and Chronic Health Evaluation- II ; CRP, C-reactive protein; ESR, erythrocyte sedimentation rate; PCT, procalcitonin; IL-2, interleukin-2; IL-6, interleukin-6; TNF- $\alpha$, tumor necrosis factor alpha; IFN- $\gamma$, interferon-gamma; LPS, lipopolysaccharide

After SARS-Cov-2 infection, the abundance of anti-inflammatory bacteria and bacteria that downregulate ACE2 expression decreases, which makes the colonization of SARS-Cov-2 in the gastrointestinal tract easier ${ }^{[3]}$. Then the GMD caused by COVID-19 severely destroys the intestinal mucosal barrier function, thus allowing the LPS and bacterial metabolites produced by the gut microbiota to easily enter the circulatory system and induce a systemic inflammatory response ${ }^{[19-21]}$. When the host immunity declines, the viral replication further accelerates, forming a "vicious cycle" that eventually leads to multiple organ failure ${ }^{[22,23]}$. WMT not only corrects the imbalance of gut microbiota effectively, but it also contributes to improvement of the intestinal mucosal barrier function and avoidance of the formation of the "vicious cycle," thus achieving an efficaciousness treatment of highly pathogenic influenza virus infection $^{[7]}$. Whether WMT exhibits its clinical efficacy in patients with COVID-19 through this proposed 
mechanism needs further investigation.

Of note, a recent systematic review on the global adverse events of FMT in the last 20 years has reported that intestinal mucosal barrier injury increases the risk of FMT-related severe adverse events, which are associated with the operation or the quality of the stool suspension $^{[24]}$. In the present trial, the physicians will be asked to respect the patient's opinion of whether to join this study and explain the risks (such as diarrhea; abdominal discomfort, pain, or cramping; nausea or vomiting; and flatulence) in detail to the patient before $\mathrm{WMT}^{[24]}$. It has been demonstrated that WMT is safer than the manual preparation of fecal microbiota ${ }^{[10]}$. Moreover, the sponsor center for providing the washed microbiota has performed WMT for years ${ }^{[25,26]}$, and only physicians with extensive experience in WMT treatment will be allowed to participate in this study.

It is stated that this trial is single-blinded. If all patients are required to be blinded to the treatment, they would need to receive an endoscopic catheterization, which is an invasive procedure that may damage the patients' health and aggravate the disease ${ }^{[27]}$. Therefore, we will abandon study blindness for the patients. However, we implement a blinding method for researchers in this study. That is, one or more staff members will be selected to be responsible for recording the clinical data and testing the experimental samples for all enrolled patients, but they will not be allowed to access the information regarding which treatment the patients have received or which group the patients belong to.

In conclusion, WMT is efficacious and safe for the treatment of COVID-19 patients suspected of having GMD. The therapeutic effects of WMT are expected to be related to improvement of the intestinal mucosal barrier function, inflammatory response, and immunity.

The protocol was preprinted on Research Square and was licensed under the $\mathrm{CC}$ by 4.0 License (DOI: 10.21203/rs.3.rs-56663/v1, URL: https://www. researchsquare.com/article/rs-56663/v1) ${ }^{[28]}$.

\section{Acknowledgments}

We thank Dr. Tao LIU for his help with the Statistical Analysis section of this article.

\section{Conflict of Interest Statement}

All authors have no conflict of interest in this study.

\section{REFERENCES}

1 Sun P, Lu X, Xu C, et al. Understanding of COVID-19 based on current evidence. J Med Virol, 2020,92(6):548551

2 Cheung KS, Hung IFN, Chan PPY, et al. Gastrointestinal manifestations of SARS-CoV-2 infection and virus load in fecal samples from a Hong Kong cohort: Systematic review and meta-analysis. Gastroenterology, 2020,159(1):81-95
3 Zuo T, Zhang F, Lui GCY, et al. Alterations in gut microbiota of patients with COVID-19 during time of hospitalization. Gastroenterology, 2020,159(3):944-955

4 Yao WU. Effect of intestinal flora imbalance on acute lung injury in mice infected with influenza virus. Modern Prevent Med (Chinese), 2018,45(4):694-699

5 Tay MZ, Poh CM, Rénia L, et al. The trinity of COVID-19: immunity, inflammation and intervention. Nat Rev Immunol, 2020,20(6):363-374

6 Bradley KC, Finsterbusch K, Schnepf D, et al. Microbiota-driven tonic interferon signals in lung stromal cells protect from influenza virus infection. Cell Rep, 2019,28(1):245-256.e244

7 Yitbarek A, Taha-Abdelaziz K, Hodgins DC, et al. Gut microbiota-mediated protection against influenza virus subtype H9N2 in chickens is associated with modulation of the innate responses. Sci Rep, 2018,8(1):13189

8 Lee PH, Tay WC, Sutjipto S, et al. Associations of viral ribonucleic acid (RNA) shedding patterns with clinical illness and immune responses in Severe Acute Respiratory Syndrome Coronavirus 2 (SARS-CoV-2) infection. Clin Transl Immunology, 2020,9(7):e1160

9 d'Ettorre G, Ceccarelli G, Marazzato M, et al. Challenges in the management of SARS-CoV2 infection: The role of oral bacteriotherapy as complementary therapeutic strategy to avoid the progression of COVID-19. Front Med (Lausanne), 2020,7:389

10 Zhang T, Lu G, Zhao Z, et al. Washed microbiota transplantation $v s$. manual fecal microbiota transplantation: clinical findings, animal studies and in vitro screening. Protein Cell, 2020,11(4):251-266

11 Nanjing consensus on methodology of washed microbiota transplantation. Chin Med J (Engl), 2020,133(19):23302332

12 National Health Commission of the People's Republic of China. COVID-19 diagnosis and treatment guideline in china, 7th ed. http://www.nhc.gov.cn/yzygj/s7653p/2 02003/46c9294a7dfe4cef80dc7f5912eb1989.shtml.

13 Lim WS, van der Eerden MM, Laing R, et al. Defining community acquired pneumonia severity on presentation to hospital: an international derivation and validation study. Thorax, 2003,58(5):377-382

14 Knaus WA, Draper EA, Wagner DP, et al. APACHE II: a severity of disease classification system. Crit Care Med, 1985,13(10):818-829

15 Ianiro G, Mullish BH, Kelly CR, et al. Screening of faecal microbiota transplant donors during the COVID-19 outbreak: suggestions for urgent updates from an international expert panel. Lancet Gastroenterol Hepatol, 2020,5(5):430-432

16 Matsumoto H, Shiotani A, Graham DY. Current and future treatment of Helicobacter pylori infections. Adv Exp Med Biol, 2019,1149:211-225

17 Thursby E, Juge N. Introduction to the human gut microbiota. Biochem J, 2017,474(11):1823-1836

18 Choi HH, Cho YS. Fecal microbiota transplantation: Current applications, effectiveness, and future perspectives. Clin Endosc, 2016,49(3):257-265

19 Li H, Liu X, Chen F, et al. Avian influenza virus subtype H9N2 affects intestinal microbiota, barrier structure injury, and inflammatory intestinal disease in the chicken ileum. Viruses, 2018,10(5):270 
20 Hanada S, Pirzadeh M, Carver KY, et al. Respiratory viral infection-induced microbiome alterations and secondary bacterial pneumonia. Front Immunol, 2018,9: 2640

21 Yildiz S, Mazel-Sanchez B, Kandasamy M, et al. Influenza A virus infection impacts systemic microbiota dynamics and causes quantitative enteric dysbiosis. Microbiome, 2018,6(1):9

22 Oldstone MB, Rosen H. Cytokine storm plays a direct role in the morbidity and mortality from influenza virus infection and is chemically treatable with a single sphingosine-1-phosphate agonist molecule. Curr Top Microbiol Immunol, 2014,378:129-147

23 Wang S, Le TQ, Kurihara N, et al. Influenza viruscytokine-protease cycle in the pathogenesis of vascular hyperpermeability in severe influenza. J Infect Dis, 2010, 202(7):991-1001

24 Marcella C, Cui B, Kelly CR, et al. Systematic review: the global incidence of faecal microbiota transplantation- related adverse events from 2000 to 2020. Aliment Pharmacol Ther, 2021,53(1):33-42

25 Ye ZN, Xia HH, Zhang R, et al. The efficacy of washed microbiota transplantation on Helicobacter pylori eradication: A pilot study. Gastroenterol Res Pract, 2020, 2020:8825189

26 Zhong HJ, Zeng HL, Cai YL, et al. Washed microbiota transplantation lowers blood pressure in patients with hypertension. Front Cell Infect Microbiol, 2021,11: 679624

27 Wang $\mathrm{S}, \mathrm{Xu} \mathrm{M}$, Wang $\mathrm{W}$, et al. Systematic review: Adverse events of fecal microbiota transplantation. PloS one. 2016,11(8):e0161174

28 He XX, Wu LH, Ye ZN, et al. Treatment of COVID-19 patients suspected with gut microbiota dysbiosis with washed microbiota transplantation: study protocol for a randomized controlled trial. Preprint at https://www. researchsquare.com/article/rs-56663/v1 (2020).

(Received Feb. 2, 2021; accepted Nov. 2, 2021) 